REFERENCES AND/OR ACKNOWLEDGEMENTS

No conflict of interest.

\section{CPS-072 COMPARISON OF IMMUNE CHECKPOINT INHIBITORS (NIVOLUMAB, PEMBROLIZUMAB, ATÉZOLIZUMAB AND DURVALUMAB) IN THE TREATMENT OF NON-SMALL CELL LUNG CANCER: TOLERANCE AND FINANCIAL IMPACT}

${ }^{1}$ F Badibouidi* ${ }^{1}$ L Dupont, ${ }^{2} \mathrm{M}$ Benchaabane, ${ }^{2} \mathrm{~S}$ Loutski. ' $G$ Ghpso, Pharmacy, Creil, France; ${ }^{2}$ Ghpso, Oncology, Creil, France

\subsection{6/ejhpharm-2020-eahpconf.173}

Background and importance Immune checkpoint inhibitors represent a major therapeutic option for the management of nonsmall cell lung cancer. However, the setback on their use in practice is limited.

Aim and objectives The aim of the study was to compare the real world data for anti-PD-1 and anti-PD-L1 antibodies (nivolumab, pembrolizumab, atézolizumab and durvalumab) in terms of tolerance and financial impact in our hospital.

Material and methods An observational study was conducted over 1 year including patients treated with either nivolumab $240 \mathrm{mg}$ or durvalumab $10 \mathrm{mg} / \mathrm{kg}$ every 2 weeks or pembrolizumab $200 \mathrm{mg}$ or atézolizumab $1200 \mathrm{mg}$ every 3 weeks.

The comparison criteria were patient profile, tolerance and cost of treatment. Annual drug costs were calculated based on VAT $(2.1 \%)$. In the case of weight dependent doses (durvalu$\mathrm{mab}$ ), mean weight was $80 \mathrm{~kg}$ (total doses per administration, $800 \mathrm{mg}$ ). The data were collected from computerised patient records (CliniCom and Chimo).

Results We analysed 53 patients: nivolumab $(n=24)$, pembrolizumab $(n=20)$, durvalumab $(n=8)$ and atézolizumab $(n=1)$. Mean age was 67 years and $79 \%$ of patients were men. The firstline treatment was durvalumab for all patients and pembrolizumab for four patients.

The mean number of treatment cycles was: nivolumab $(n=16)$, pembrolizumab $(n=9.3)$, durvalumab $(n=4.5)$ and atézolizumab $(n=6)$. Side effects occurred in $64 \%$ of patients (79\% nivolumab, 45\% pembrolizumab and 50\% durvalumab). Haemoptysis caused hospitalisation in two patients (pembrolizumab $\mathrm{n}=1$, durvalumab $\mathrm{n}=1$ ). Reasons for stopping treatment were progression (9\% nivolumab, 25\% pembrolizumab and $100 \%$ atézolizumab) and side effects (14\% nivolumab, $15 \%$ pembrolizumab and $12.5 \%$ durvalumab). The most common side effects were pneumonitis (37\% nivolumab and 5\% pembrolizumab), metabolism disorders $(25 \%$ durvalumab, $12.5 \%$ nivolumab and $5 \%$ pembrolizumab) and diarrhoea (15\% pembrolizumab and $8 \%$ nivolumab). The annual costs of treatments were $€ 61871$ for atézolizumab, $€ 66000$ for nivolumab, $€ 93038$ for pembrolizumab and $€ 100450$ durvalumab.

Conclusion and relevance Our study showed that the incidence of pneumonitis seemed to be higher with nivolumab and that treatment interruption was more important for pembrolizumab. Nivolumab seemed to be generally better tolerated than the other agents. Nevertheless, for patients with baseline respiratory diseases, pembrolizumab could be considered the preferred option.

\section{REFERENCES AND/OR ACKNOWLEDGEMENTS}

1. Giuliani J, et al. Anticancer Res 2019;39:3961-3965.

\section{CPS-073 EFFECTIVENESS AND SAFETY OF PLATIN/ PERMETREXED COMBINATION IN NON-SMALL CELL LUNG CANCER}

EM Barreiro Fernandez* , C Martinez-Diaz, FJ Salmeron-Navas, M Dominguez-Cantero, S Fenix-Caballero. Hospital Universitario Puerto Real, Servicio De Farmacia, Cadiz, Spain

\subsection{6/ejhpharm-2020-eahpconf.174}

Background and importance According to the PARAMOUNT trial, induction chemotherapy with a platin/pemetrexed combination and pemetrexed maintenance therapy reduced the risk of progression free survival (PFS) and overall survival (OS) in patients with non-cell lung cancer (NSCLC).

Aim and objectives The aim of the study was to assess the effectiveness and safety of this drug combination in NSCLC and to evaluate the degree of agreement with the PARAMOUNT results.

Material and methods A descriptive retrospective study was conducted. All patients that initiated treatment with platin/ pemetrexed from January 2018 to September 2019 were included. Clinical data were obtained from digital clinical history and the prescription software Farmis Oncofarm: sex, age, stage, performance status (PS), periodicity of chemotherapy, dose received and number of cycles. PFS and OS were used as efficacy end points, and were obtained by the KaplanMeier method (SPSS Statistics programme).

In terms of safety, adverse events (AE) of any grade were recorded for assessment of the safety profile. Effectiveness data and safety were compared with the PARAMOUNT results.

Results Forty-two patients were enrolled, 36 men and 6 women, with an average age of 67 years (range 42-80). Cancer stage was as follows: stage IV (90\%), stage IIB (7\%) and stage IIIA (3\%). Baseline PS was $0-1$ in $60 \%$ of cases and in the remainder, 2-3. All patients received as induction therapy on day 1, 21 day cycles of pemetrexed $\left(500 \mathrm{mg} / \mathrm{m}^{2}\right)$ in combination with cisplatin $75 \mathrm{mg} / \mathrm{m}^{2} \quad(\mathrm{n}=16)$ or carboplatin AUC $=5 \quad(n=26)$. Pemetrexed maintenance therapy $(500 \mathrm{mg} /$ $\mathrm{m}^{2}$ ) was administrated until progression or death. The median number of cycles was 4 (1-16). Median PFS was 4 months (95\% CI 3 to 5) and median OS was 17 months (95\% CI 11 to 21). In the PARAMOUNT study, median PFS was 4 months and median OS was 14 months. Sixty per cent of patients $(n=25)$ had AE. The most common AE were mucositis $(n=7)$, asthenia $(n=6)$, diarrhoea $(n=3)$, dermatitis $(n=3)$, vomiting $(n=3)$, anaemia $(n=2)$ and neutropenia $(n=2)$. In the clinical trial, the most common $\mathrm{AE}$ of any grade were anaemia, neutropenia, fatigue and nausea.

Conclusion and relevance PFS and OS showed a clinical benefit. The safety profile for the use of this combination showed it was tolerated. The effectiveness and AE were similar compared with the published clinical trial.

\section{REFERENCES AND/OR ACKNOWLEDGEMENTS}

No conflict of interest.

\section{CPS-074 CYCLIN DEPENDENT KINASE 4/6 INHIBITORS IN BREAST CANCER: POTENTIAL DRUG INTERACTIONS}

${ }^{1}$ MI Bernias Domínguez*, 'M Mañes Sevilla, ${ }^{2} \mathrm{PM}$ Bernias Domínguez, ${ }^{1} \mathrm{MJ}$ Vazquez Castillo, ${ }^{1} \mathrm{C}$ Moriel Sánchez. ${ }^{1}$ Hospital Universitario De Móstoles, Hospital Pharmacy, Madrid, Spain; ${ }^{2}$ Fremap, Medicine, Madrid, Spain

10.1136/ejhpharm-2020-eahpconf.175 
Background and importance Selective cyclin dependent kinase (CDK) inhibitors, palbociclib and ribociclib, were recently approved to treat advanced or metastatic breast cancer. The hospital pharmacist plays an important role in the revision of the treatment at consultation, in order to ensure the safety and effectiveness of the treatment.

Aim and objectives To analyse potential drug interactions (PDI) before starting palbociclib or ribociclib treatment and to evaluate physician acceptance of pharmacist recommendations.

Material and methods This was a retrospective observational study including all patients who started treatment with palbociclib or ribociclib in a second level hospital until September 2019. At the beginning of treatment, the pharmacist interviewed patients and reviewed their medication in the pharmaceutical consultation. All PDI detected were analysed, making an intervention as therapeutic recommendations.

PDI were identified using Lexicomp, Stockley's Drug Interactions, Micromedex and CheckTheMeds. PDI were classified as moderate (pharmacological effects must be controlled) or severe (drug combination should be avoided). Follow-up of the recommendations was made 1 month after the beginning of treatment at the pharmaceutical consultation.

Results Twenty-eight patients started palbociclib (50\%) or ribociclib $(50 \%)$ treatment in our hospital $(95.9 \%$ women; mean age $63.6 \pm 9.8$ years). Sixteen (57\%) were polymedicated; the average number of medications per patient (not including endocrine and CDK inhibitors therapy) was 6.25. Thirty-one PDI were detected in 18 different patients (64.3\%). There were $14(45.2 \%)$ severe PDI and 17 (54.8\%) moderate PDI. The most common types of drugs involved were statins (22.6\%), proton pump inhibitors (22.6\%), antidepressants (12.9\%) and pyrazolones (16.1\%).

Eleven severe PDIs were accepted (78.6\%). Moderate recommendations led to a reduction in antidepressant dosage $(5.9 \%)$ and two change of drugs involved in the interaction (11.8\%).

Conclusion and relevance This study showed that more than half of patients that started treatment with CDK inhibitors has at least one PDI. Clinical pharmacists are essentials in detecting PDI, which is a positive influence on physician prescriptions and patient treatment outcomes, improving the safety and effectiveness of the oncological treatment.

\section{REFERENCES AND/OR ACKNOWLEDGEMENTS}

No conflict of interest.

\section{CPS-075 IBRUTINIB ASSOCIATED ATRIAL FIBRILLATION: INCIDENCE AND MANAGEMENT IN THE REAL LIFE CLINICAL SETTING}

S Blanco-Dorado*, A Mosquera-Torre, E López-Montero, M Touris-Lores, B Bernardez Ferran, I Zarra-Ferro. Complejo Hospitalario Universitario De Santiago De Compostela, Pharmacy Department, Santiago De Compostela, Spain

\subsection{6/ejhpharm-2020-eahpconf.176}

Background and importance Atrial fibrillation (AF) is one of the most common side effects of ibrutinib, a drug that has improved the prognosis of chronic $\mathrm{B}$ cell malignancies. The incidence of ibrutinib related AF (IRAF) is not well known in the 'real life' setting, and management is challenging, especially due to the risk of bleeding with ibrutinib and its pharmacological interactions with antiarrhythmics and anticoagulants.
Aim and objectives To determine the incidence of IRAF, and to analyse the characteristics and treatment of this arrhythmia in a real life clinical setting.

Material and methods A retrospective observational study was conducted including patients treated with ibrutinib. Patient characteristics and the management of IRAF were recorded using the electronic medical history. Numerical variables were expressed as mean (SD) and categorical as frequencies (percentages).

Results Twenty-eight patients were treated with ibrutinib and $5 / 28(17.8 \%)$ patients developed IRAF. Patient characteristics are shown in table 1 .

\begin{tabular}{lll}
\multicolumn{2}{l}{ Abstract 4CPS-075 } & Table 1 \\
& $\begin{array}{l}\text { Patients without IRAF=23 } \\
(85.2 \%)\end{array}$ & $\begin{array}{l}\text { Patients with IRAFN=5 } \\
(17.8 \%)\end{array}$ \\
\hline Age (years) & $73 \pm 11$ & $75 \pm 6$ \\
Sex (M/F) & $12 / 11$ & $4 / 1$ \\
Previously AF (n (\%)) & $1(4.4)$ & $1(20)$ \\
Indication LLC ( $(\%))$ & $20(86.9)$ & $4(80.0)$ \\
Ibrutinib duration & $13.7 \pm 11.2$ & $18.6 \pm 9.7$ \\
(months) & &
\end{tabular}

Of the 5 patients who developed IRAF, 2 were grade 3 requiring electric cardioversion and discontinuation of treatment until recovery to grade 1 . The other three cases were grade 1 or 2 and treatment was not suspended. In all 5 patients, anticoagulant was initiated (apixaban in 3, rivaroxaban and low molecular weight heparin in 1 patient, respectively). Treatment with beta-blockers was started in 3 patients and in 1 patient the arrhythmia was recurrent, requiring new cardioversion, initiation of amiodarone treatment and ibrutinb dose adjustment. Median time for the appearance of IRAF was 13 months. No major bleeding events occurred.

Conclusion and relevance This study showed a higher prevalence of IRAF similar to other studies in real life, but with a longer median onset, justifying close monitoring during the first months but also throughout treatment with ibrutinib.

\section{REFERENCES AND/OR ACKNOWLEDGEMENTS}

1. Stephens DM, Byrd JC. How I manage ibrutinib intolerance and complications in patients with chronic lymphocytic leukemia. Blood 2019;133:1298-1307. doi: 10.1182/blood-2018-11-846808. Epub 2019 January 14.

No conflict of interest.

\section{CPS-076 INDIRECT COMPARISON BETWEEN PEMBROLIZUMAB MONOTHERAPY AND PEMBROLIZUMAB CHEMOTHERAPY REGIMENS IN SQUAMOUS LUNG CANCER}

MDP Briceño Casado*, MD Gil-Sierra, S Fenix-Caballero, M Dominguez-Cantero, EJ AlegreDel Rey. Hospital Universitario Puerto Real, Hospital Pharmacy, Cadiz, Spain

\subsection{6/ejhpharm-2020-eahpconf.177}

Background and importance Pembrolizumab monotherapy $(\mathrm{Pb})$ showed benefit in terms of overall survival (OS) and progression free survival (PFS) compared with chemotherapy alone $(\mathrm{CT})$ in untreated metastatic non-small cell lung cancer (mNSCLC) with PD-L1 $\geq 50 \%$ expression. The Pb-CT 